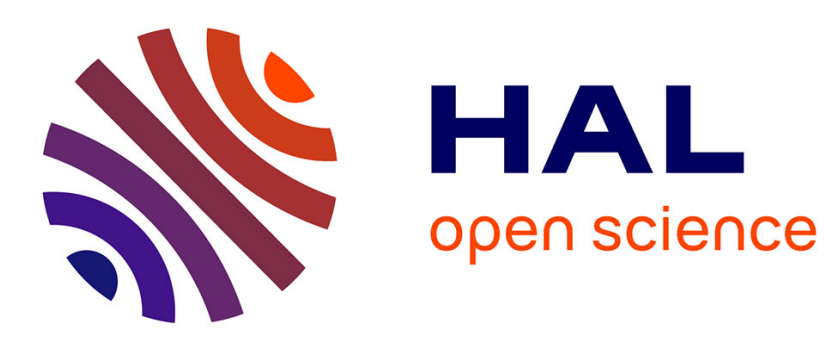

\title{
Mathematical Modeling of Metabolism and Hemodynamics
}

R. Costalat, Jean-Pierre Françoise, C. Menuel, M. Lahutte, J. Vallée, Giovanni de Marco, J. Chiras, Rémy Guillevin

\section{- To cite this version:}

R. Costalat, Jean-Pierre Françoise, C. Menuel, M. Lahutte, J. Vallée, et al.. Mathematical Modeling of Metabolism and Hemodynamics. Acta Biotheoretica, 2012, 60 (1-2), pp.99 - 107. 10.1007/s10441012-9157-1 . hal-01406297

\section{HAL Id: hal-01406297 https://hal.sorbonne-universite.fr/hal-01406297}

Submitted on 10 Oct 2019

HAL is a multi-disciplinary open access archive for the deposit and dissemination of scientific research documents, whether they are published or not. The documents may come from teaching and research institutions in France or abroad, or from public or private research centers.
L'archive ouverte pluridisciplinaire HAL, est destinée au dépôt et à la diffusion de documents scientifiques de niveau recherche, publiés ou non, émanant des établissements d'enseignement et de recherche français ou étrangers, des laboratoires publics ou privés. 


\title{
Mathematical modeling of metabolism and hemodynamics
}

\author{
R. Costalat · J.-P. Francoise - C. Menuel · M. \\ Lahutte · J.-N. Vallée · G. de Marco · J. \\ Chiras · R. Guillevin
}

\begin{abstract}
We provide a mathematical study of a model of energy metabolism and hemodynamics of glioma allowing a better understanding of metabolic modifications leading to anaplastic transformation from low grade glioma.
\end{abstract}

Keywords Magnetic resonance spectroscopy $\cdot$ multinuclear spectroscopy $\cdot$ regional cerebral blood flow $\cdot$ differential equation $\cdot$ viability $\cdot$ slow-fast dynamics

Partially supported by an ANR grant "Analyse non linéaire et applications aux rythmes du vivant" BLAN07 - 2- 182920 .

R. Costalat

UPMC, UMI 209, UMMISCO, University of Paris-6, 75005 Paris, France, IRD, UMI 209, UMMISCO,

93143 Bondy cedex, France

J.-P. Francoise

Université P.-M. Curie, Paris 6, Laboratoire Jacques-Louis Lions, UMR 7598 CNRS, 4 Pl. Jussieu, 16-26, 75252 Paris, France

C. Menuel

Inserm U678, Functional Imaging Laboratory, Department of Neuroradiology, Pitiè-Sapêtrière Hospital, Université P.-M. Curie, Paris 6, 4783, boulevard de l'Hôpital,75651 Paris cedex 13, France

M. Lahutte

Hopital des Armées, Val-de-Grâce, Service de Radiologie, 74 Bd de Port-Royal, 75005 Paris, France

J.-N. Vallé

Department of Neuroradiology, Amiens University Medical Center, University of Picardie-Jules Vernes, chemin du Thil, 80025 Amiens, France

G. de Marco

Laboratoire contrôle moteur et mouvement, UFR STAPS, Paris X, 200, avenue de la République, 92001 Nanterre, France

J. Chiras

Inserm U678, Functional Imaging Laboratory, Department of Neuroradiology, Pitiè-Sapêtrière Hospital, Université P.-M. Curie, Paris 6, 4783, boulevard de l'Hôpital,75651 Paris cedex 13, France

R. Guillevin

Inserm U678, Functional Imaging Laboratory, Department of Neuroradiology, Pitiè-Sapêtrière Hospital, Université P.-M. Curie, Paris 6, 4783, boulevard de l’Hôpital,75651 Paris cedex 13, France 
PACS PACS code $1 \cdot$ PACS code $2 \cdot$ more

Mathematics Subject Classification (2000) MSC 34C05 · MSC 34A34 · 34C14

\section{Introduction}

Because anaplastic transformation of low grade glioma is inescapable and occurs in variable delay, their therapeutic management appears to be a major issue. Until now, clinical and imaging data failed to promote predictive arguments. Only serial volumetric determination may give informations on further evolution, but with a timescale of one year or more. On the other hand, metabolic changes linked to anaplastic transformation occur with smaller timescale. Thus, metabolic glioma investigation may provide informations on further histological and morphological modifications of glioma. Finally, therapeutic management may be influenced by these issues. Multinuclear magnetic resonance spectroscopy ${ }^{1} \mathrm{H} /{ }^{31} \mathrm{P}$ allows non invasive follow-up of brain tumors metabolism. Metabolites concentrations measurements as creatine and phosphocreatine, lactate, $A T P$, intracellular $p H$ can be determined by this technique. In addition, regional cerebral blood flow $(r C B F)$ can be obtained during the same time of examination. Robustness and relevance of these tools have been previously established [4], [7]. However, metabolic and hemodynamic modifications underlying glioma evolution did not receive attention before. With the aim of better understanding pathophysiological mechanisms in this field, we built a mathematical model. In a previous paper [5], we derived a system of ordinary differential equations. This model was based on previously published physiological models [1], [2]. On the basis of this model, we suggested that specific profiles of metabolic changes may constitute an early indicator of further anaplastic transformation. This prompted us to further study the mathematical properties of the model, and this is the aim of this paper. We intent to determine whether non trivial mathematical properties of the model can give useful pathophysiological indications for better management of glioma.

\section{Derivation of the Model}

The model is inspired by the analysis of brain lactate metabolism developed in [1], [2]. For sake of simplicity, the molar concentration of species $X$, namely $[X]$, is noted $X$; units are $m M$. In this model the two state variables are the intracapillary lactate concentration $L A C_{c}$ and the intracellular lactate concentration $L A C_{i}$. We assume that the total volume of neurons and normal glial cells is smaller than glioma volume within the volume of interest, so that the subscript " $i$ " refers to the intracellular milieu of glioma cells; similarly, "cells" designate glioma cells. The model includes the following elements (cf Fig. 1):

(i) intracellular lactate concentration $L A C_{i}$ and $p H\left(p H_{i}=-\log H_{i}^{+}\right)$, cell volume being $V_{i}$;

(ii) cell lactate production $J_{1}$;

(iii) flux of lactate diffusion from cells to capillaries $J_{2}$;

(iv) flux $J_{3}$, which is the sum of : 
- lactate consumption by the metabolism, taking into account both the conversion lactate-pyruvate catalysed by lactate dehydrogenase and subsequent consumption of pyruvate by mitochondria, and a possible consumption of lactate by remaining neurons (astrocyte-neuron lactate shuttle, as proposed by [9],

- lactate diffusion towards neighbouring regions;

(v) capillary lactate concentration $\left(L A C_{c}\right)$ and $p H\left(p H_{c}=-\log H_{c}^{+}\right)$, capillary volume being $V_{c}$;

(vi) arterial lactate concentration $L A C_{a}$;

(vii) cerebral blood flow $(C B F)$;

(viii) flux $J_{\text {cap }}$, which is the difference between lactate input to capillaries and output from capillaries, namely $J_{c a p}=C B F . L A C_{a}-C B F . L A C_{v}$, where $L A C_{v}$ is the venous lactate concentration.

Furthermore, volumes and blood flow values are expressed per unit tissue volume. As a consequence, $V_{c}$ and $V_{i}$ are dimensionless parameters, and the capillary blood flow $C B F$ is expressed in $s^{-1}$.

Thus the following mass balance equations can readily be obtained:

$$
V_{i} \frac{d L A C_{i}}{d t}=J_{1}-J_{2}-J_{3}, \quad V_{c} \frac{d L A C_{c}}{d t}=J_{c a p}+J_{2} .
$$

Constitutive equations were derived in the following way. First, transport of lactate is always coupled to $\mathrm{H}^{+}$transport via monocarboxylate transporters $(M C T s)$. One can simply take into account this passive co-transport by setting (Aubert et al. 2005):

$$
J_{2}=T\left(\frac{L A C_{i} H_{i}^{+}}{K_{H}+L A C_{i} H_{i}^{+}}-\frac{L A C_{c} H_{c}^{+}}{K_{H}+L A C_{c} H_{c}^{+}}\right),
$$

where $K_{H}$ is a constant expressed in $m M . M, T$ the maximum transport rate. This formula is a simplified version of a more general equation for carrier-mediated symport [6].

Furthermore, following a suggestion by [3] for oxygen balance, we set:

$$
J_{\text {cap }}=C B F \cdot L A C_{a}-C B F \cdot L A C_{v}=2 C B F\left(L A C_{a}-L A C_{c}\right) .
$$

In previous papers, we showed that this simple formulation is nearly equivalent to more complex ones, based on partial differential equations [11], and can be applied to lactate, glucose, and oxygen in brain [1], [2]. Finally, since $J_{1}$ and $J_{3}$ respective contributions are difficult to distinguish on the basis of clinical magnetic resonance studies, we simply write $J=J_{1}-J_{3}$.

Finally, we obtain the following system of two differential equations:

$$
\begin{gathered}
V_{i} \frac{d L A C_{i}}{d t}=J-T\left(\frac{L A C_{i} H_{i}^{+}}{K_{H}+L A C_{i} H_{i}^{+}}-\frac{L A C_{c} H_{c}^{+}}{K_{H}+L A C_{c} H_{c}^{+}}\right), \\
V_{c} \frac{d L A C_{c}}{d t}=2 C B F .\left(L A C_{a}-L A C_{c}\right)+T\left(\frac{L A C_{i} H_{i}^{+}}{K_{H}+L A C_{i} H_{i}^{+}}-\frac{L A C_{c} H_{c}^{+}}{K_{H}+L A C_{c} H_{c}^{+}}\right) .
\end{gathered}
$$

It must be noted that the capillary volume $V_{c}$ is much smaller than the cell volume $V_{i}$; typically, $V_{c} / V_{i}$ is about 0.01 . Setting $\varepsilon=V_{c} / V_{i}$ and $\tau=t / V_{i}$, we can write: 


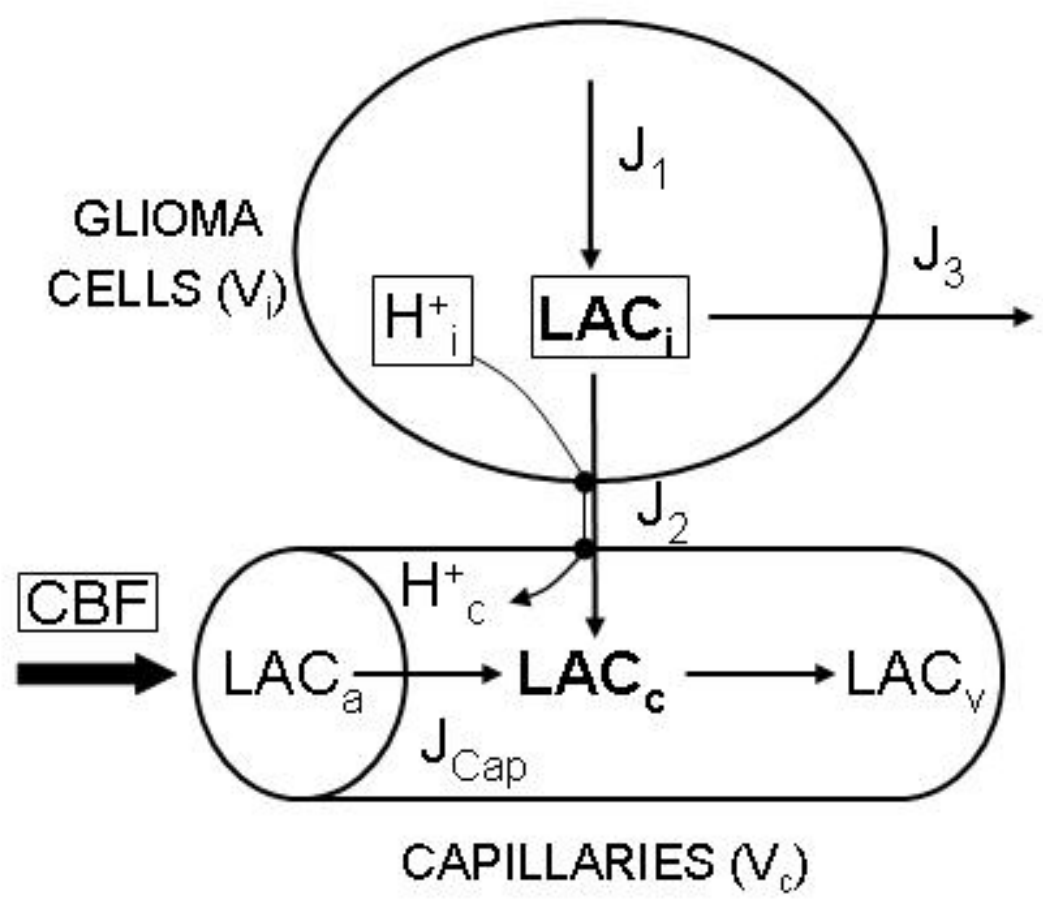

Fig 1 


$$
\begin{gathered}
\frac{d L A C_{i}}{d \tau}=J-T\left(\frac{L A C_{i} H_{i}^{+}}{K_{H}+L A C_{i} H_{i}^{+}}-\frac{L A C_{c} H_{c}^{+}}{K_{H}+L A C_{c} H_{c}^{+}}\right), \\
\varepsilon \frac{d L A C_{c}}{d \tau}=2 C B F .\left(L A C_{a}-L A C_{c}\right)+T\left(\frac{L A C_{i} H_{i}^{+}}{K_{H}+L A C_{i} H_{i}^{+}}-\frac{L A C_{c} H_{c}^{+}}{K_{H}+L A C_{c} H_{c}^{+}}\right) .
\end{gathered}
$$

In the following mathematical study (paragraphs 3-5), we write $t$ instead of $\tau$, bearing in mind that a moderate change in time scale has been achieved by introducing $\tau$ since $V_{i}$ value is about 0.5 .

\section{Phase-plane analysis of the system with $J$ and $T$ constant}

We change to notations better adapted to the mathematical analysis. We set:

$$
x=L A C_{i}, y=L A C_{c}, k=K_{H} / H_{i}^{+}, k^{\prime}=K_{H} / H_{c}^{+}, L=L A C_{a}, F=2 C B F .
$$

We thus obtain the fast-slow system:

$$
\begin{gathered}
\frac{d x}{d t}=J-T\left(\frac{x}{k+x}-\frac{y}{k^{\prime}+y}\right) \\
\varepsilon \frac{d y}{d t}=F(L-y)+T\left(\frac{x}{k+x}-\frac{y}{k^{\prime}+y}\right) .
\end{gathered}
$$

In this first paragraph, we consider $F, L, k, k^{\prime}$ as fixed and $J, T$ as parameters. We discuss the existence and nature of the stationnary point as well as eventual existence of periodic orbits. But we also decide that the system makes only sense in a fixed rectangle that we call the viable phase space:

$$
V=\{(x, y), 0 \leq x \leq M, 0 \leq y \leq N\}
$$

This is motivated by the fact that the variables $x$ and $y$ must be positive as they represent concentrations and cannot assume very large values. We say that if an orbit leaves the domain $V$ it is not viable. Our interpretation is that the biological viability of the system is no longer ensured, e.g. cell necrosis occurs.

\subsection{Stationary points}

Solving the system:

$$
\begin{gathered}
0=J-T\left(\frac{x}{k+x}-\frac{y}{k^{\prime}+y}\right) \\
0=F(L-y)+T\left(\frac{x}{k+x}-\frac{y}{k^{\prime}+y}\right),
\end{gathered}
$$

yields

$$
y=L+\frac{J}{F}=y_{0}
$$


which is always positive. This displays:

$$
x=\frac{k\left(\frac{J}{T}+\frac{y_{0}}{k^{\prime}+y_{0}}\right)}{1-\left(\frac{J}{T}+\frac{y_{0}}{k^{\prime}+y_{0}}\right)}=x_{0} .
$$

There is, thus, a unique stationary point $\left(x_{0}, y_{0}\right)$.

3.2 Nature of the stationary point

The nature of the stationary point $\left(x_{0}, y_{0}\right)$ can be discussed on the Jacobian of the system at this point. The eigenvalues $\lambda_{ \pm}$solve the equation:

$$
(A+\lambda)\left(\frac{B+F}{\varepsilon}+\lambda\right)-\frac{A B}{\varepsilon}=0,
$$

with

$$
A=\frac{k T}{(k+x)^{2}}, B=\frac{k^{\prime} T}{\left(k^{\prime}+y\right)^{2}} .
$$

The eigenvalues are so that:

$$
\begin{gathered}
\lambda_{+}+\lambda_{-}=-\left(A+\frac{B+F}{\varepsilon}\right)<0, \\
\lambda_{+} \lambda_{-}=A F / \varepsilon>0,
\end{gathered}
$$

hence the stationary point is stable. Furthermore,

$$
\begin{gathered}
\Delta=\left(A+\frac{B+F}{\varepsilon}\right)^{2}-4 \frac{A F}{\varepsilon}= \\
A^{2}+2 A\left(\frac{B+F}{\varepsilon}\right)+\left(\frac{B+F}{\varepsilon}\right)^{2}-4 \frac{A F}{\varepsilon}> \\
A^{2}-2 A\left(\frac{B+F}{\varepsilon}\right)+\left(\frac{B+F}{\varepsilon}\right)^{2} \geq 0,
\end{gathered}
$$

hence this unique stationary point is a node.

\section{Control of the position of the stationary point}

We aim in this section to write explicitly the conditions on the control $(J, T)$ and the parameters so that:

$$
0 \leq y_{0} \leq M, 0 \leq x_{0} \leq N .
$$

It was observed previously that $y_{0}$ is always positive. But there is a condition so that $x_{0}>0$. This yields

$$
1-\left(\frac{J}{T}+\frac{y_{0}}{k^{\prime}+y_{0}}\right)>0,
$$

with

$$
y_{0}=L+\frac{J}{F},
$$


this displays the condition

$$
T>J\left[1+\frac{1}{k^{\prime}}\left(L+\frac{J}{F}\right)\right] .
$$

This shows, in particular that the condition $T>J$ is necessary.

The condition $y_{0} \leq M$ displays:

$$
\frac{J}{F} \leq(M-L)
$$

Note that the rectangle should be such that $M \geq L$. The last condition

$$
x_{0} \leq N
$$

yields:

$$
\frac{y_{0}}{k^{\prime}+y_{0}}<\frac{N}{k+N}-\frac{J}{T}
$$

and

$$
L+\frac{J}{F}<\frac{k^{\prime}\left(\frac{N}{k+N}-\frac{J}{T}\right)}{\frac{k}{k+N}+\frac{J}{T}} .
$$

\section{The slow curve}

The geometry of the slow curve is important as it allows to explain how the orbits may eventually leave the viability domain.

The equation of the slow curve is:

$$
f(x, y)=F(L-y)+T\left(\frac{x}{k+x}-\frac{y}{k^{\prime}+y}\right)=0 .
$$

This slow curve is always attractive because:

$$
f_{y}^{\prime}(x, y)=-F-\frac{k^{\prime} T}{\left(k^{\prime}+y\right)^{2}}<0 .
$$

Note as well that it is a graph over the $y$-axis: $f(x, y)=0$ if and only if $x=\phi(y)$, with:

$$
x=\frac{k\left(F y-F L+\frac{T y}{k^{\prime}+y}\right)}{T-\left(F y-F L+\frac{T y}{k^{\prime}+y}\right)} .
$$

The function $\phi$ is increasing. Generic orbits are almost parallel to the $y$-axis then reach a neighborhood of the slow curve and follow inside this neighborhood untill they tend to the stationary point.

Numerical computations using MATLAB software clearly confirm this point: orbits in the $(x, y)=\left(L A C_{i}, L A C_{c}\right)$ phase plane are displayed in Figure 2, where $\varepsilon=V_{c} / V_{i}=0.006875$. Moreover, even values of $\varepsilon$ as high as 1 result in a somewhat similar phase portrait (not shown). 


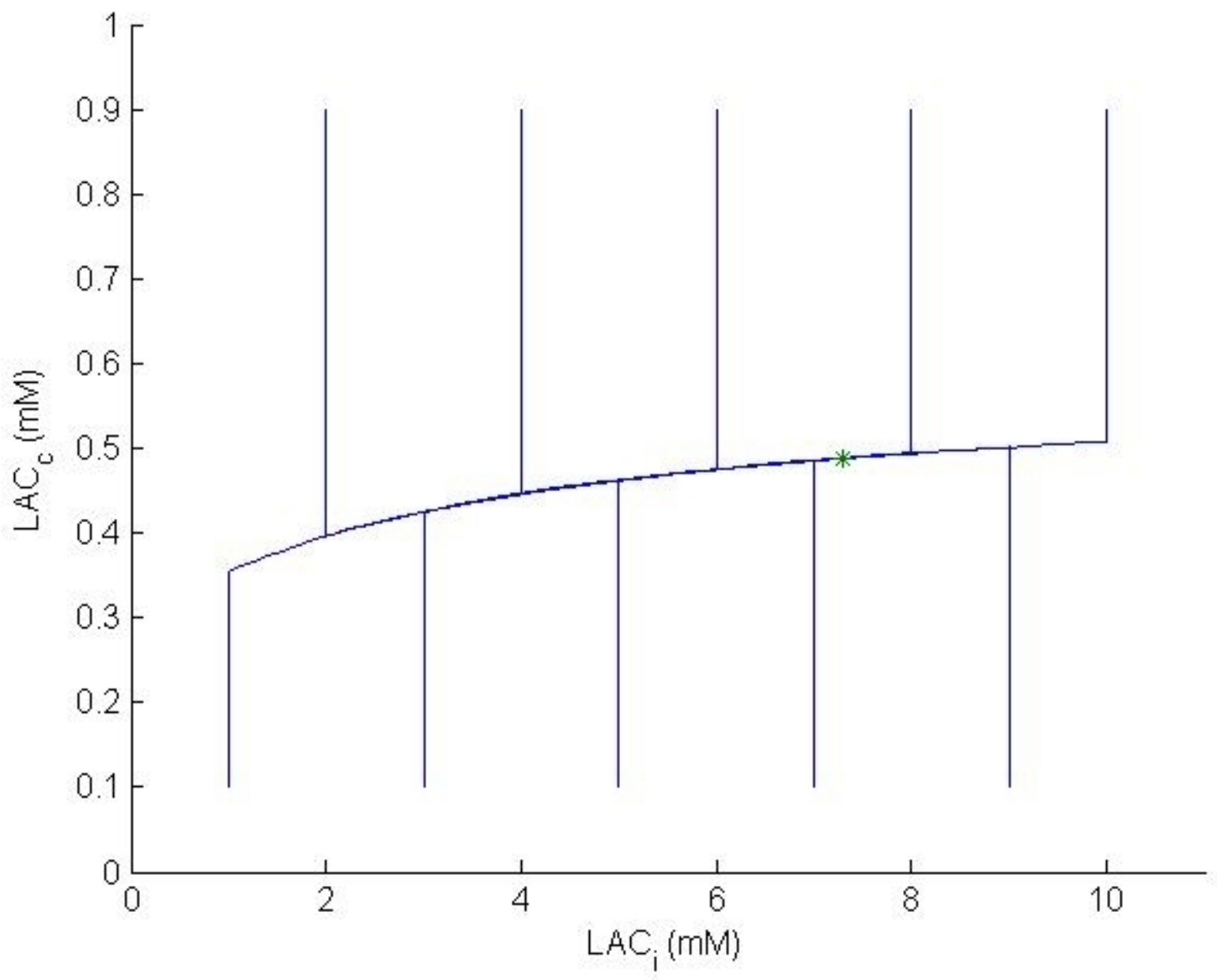

Fig. 2 Please write your figure caption here

\section{Discussion and Conclusion}

Whatever the parameters values, the model has a unique stationary point. Furthermore, the stationary point is asymptotically stable. This finding is consistent with a clinically observed fact that, within a short time scale from minutes to days, metabolite concentrations within the tumor appear nearly constant. Moreover, we derived explicit and sufficient conditions which ensure that a stationary point is in a viability domain in the first quadrant. These conditions can give useful pathophysiological insights on tumor viability. For instance, " $x(t)$ is positive" implies that $T$, the maximum transport of lactate via $M C T$, must be sufficiently high. This strongly suggests modifications of density and /or kinetic properties of $M C T$ during glioma evolution. In 
fact, both $M C T$ in glioma cells and blood-brain barrier should have enhanced density or individual maximal rate. This also suggests that $M C T$ could be potential target for glioma therapeutics [8]. Furthermore, the two variables of the system display distinct time evolutions. Thus, the system could be studied using asymptotic and geometric analysis of slow-fast systems. Quite interestingly, the model has an associated viability domain, and generic orbits are almost parallel to the $Y\left(L A C_{c}\right)$ axis, then remain in neighborhood of the slow curve while tending to the stationary point. As a consequence, generic orbits do not leave the viability domain. Thus, in the framework of the slow-fast dynamics approximation, the problem of the viability of trajectories (solution curves) can fully be solved. This will allow using a larger study frame where some parameters of the model can be replaced by control variables. There is indeed the perspective to build a hierarchy of models for cerebral metabolism and hemodynamics, in analogy with the SAPHIR model built for renal physiology [10] by replacing successively each control variables by a compartmental adding of supplementary models, on the basis of the Aubert-Costalat equations. As a conclusion, the model provides pathophysiological mechanisms of glioma metabolism. Further mathematical and clinical studies can provide a better understanding of the natural history of glioma and then may allow improvement of therapeutic management.

\section{References}

1. A. Aubert and R. Costalat, Interaction between astrocytes and neurons studied using a mathematical model of compartmentalized energy metabolism, J Cereb Blood Flow Metab, 25, 1476-1490, (2005)

2. A. Aubert, R. Costalat, P.-J. Magistretti and L. Pellerin, Brain lactate kinetics: Modeling evidence for neuronal lactate uptake upon activation, Proc. Nat. Ac. Sci., vol. 102 45, 16448-16453, (2005)

3. Gjedde A, Cerebrovascular disease, 23-40, Batjer(Ed) Lippincott-Raven, Philadelphia, (1997)

4. Guillevin R. et al., Proton magnetic resonance spectroscopy predicts proliferative activity in diffuse low-grade gliomas, J. Neurooncol., 87, 181-187, (2008).

5. Guillevin R., et al., Mathematical modeling of energy metabolism and hemodynamics of WHO grade II gliomas using in vivo MR data, CR Biologies,334:1, 31-38, (2011)

6. Keener J and Sneyd J, Mathematical physiology. Springer-Verlag, New-York, (1998).

7. Law $\mathrm{M}$ et al., Perfusion magnetic resonance imaging predicts patient outcome as an adjunct to histopathology: a second reference standard in the surgical and nonsurgical treatment of low-grade gliomas, Neurosurgery, 58(6), 1099-1107, (2006)

8. Mathupala SP, Parajuli P and Sloan AE, Silencing of monocarboxylate transporters via small interfering ribonucleic acid inhibits glycolysis and induces cell death in malignant glioma: an in vitro study, Neurosurgery 55, 1410-1419, (2004)

9. Pellerin L and Magistretti PJ, Glutamate uptake into astrocytes stimulates aerobic glycolysis: A mechanism coupling neuronal activity to glucose utilization, Proc Natl Acad Sci USA, 91, 10625-10629, (1994)

10. Thomas, S.R. et al., SAPHIR: a physiome core model of body fluid homeostasis and blood pressure regulation, Philos. Trans. A Math. Phys. Eng. Sci., 366, 3175-3197, (2008)

11. Valabrègue $\mathrm{R}$ et al., Relation between cerebral blood flow and metabolism explained by a model of oxygen exchange, J Cereb Blood Flow Metab, 23, 536-545, (2003) 